\title{
A 5-day cytoreductive chemotherapy followed by haplo-identical hsct (FA5-BUCY) as a tumor-ablative regimen improved the survival of patients with advanced hematological malignancies
}

\author{
Ting Yang ${ }^{1, *}$, Qiaoxian Lin ${ }^{1, *}$, Jinhua Ren ${ }^{1, *}$, Ping Chen $^{1}$, Xiaohong Yuan ${ }^{1}$, Xiaofeng \\ Luo ${ }^{1}$, Tingbo Liu ${ }^{1}$, Jing Zheng ${ }^{1}$, Zhihong Zheng ${ }^{1}$, Xiaoyun Zheng ${ }^{1}$, Xinji Chen ${ }^{1}$, \\ Langhui Zhang ${ }^{1}$, Hao Zheng ${ }^{1}$, Zaisheng Chen ${ }^{1}$, Xueling Hua ${ }^{1}$, Shaohua Le ${ }^{1}$, Jian \\ $\mathbf{L i}^{1}$, Zhizhe Chen ${ }^{1}$, Jianda $\mathrm{Hu}^{1}$ \\ ${ }^{1}$ Department of Hematology, Fujian Institute of Hematology, Fujian Provincial Key Laboratory on Hematology, Fujian Medical \\ University Union Hospital, Fuzhou 350001, Fujian, P. R. China \\ "These authors have contributed equally to this work \\ Correspondence to: Jianda Hu, email: drjiandahu@163.com \\ Keywords: haplo-identical HSCT, cytoreductive chemotherapy, refractory, hematological malignancies, disease control \\ Received: June 11,2016 Accepted: September 20,2016 Published: October 1, 2016
}

\section{ABSTRACT}

Haplo-HSCT has been used when HLA-matched siblings are not available. Conditioning regimens aim to reduce tumor burden prior to HSCT and provide sufficient immunoablation. We report the outcome of haplo-HSCT in 63 consecutive patients from 2/2013 to 12/2015 (19 females/44 males) with high-risk or relapsed/ refractory hematological malignancies ( $n=29-A M L ; 8-s A M L ; 19-A L L ; 5$-advancedMDS; 2-CML-BC). Median age was 20 years (range: 1.1-49). Twenty-one patients achieved remission prior to transplant, while 42 did not. Patients received FA5-BUCY, i.e., 5-day salvage chemotherapy (Fludarabine/Ara-C) and conditioning (Busulfan/ Cyclophosphamide). GvHD prophylaxis included ATG, CsA, MMF and short-term MTX. All patients received stem cells from bone marrow and peripheral blood, and achieved successful engraftment, except two who died before. With a median follow-up of 269 days (120-1081), 42/63 patients are still alive and disease-free. Two-year OS and RFS were similar in patients not in remission and in those in complete remission $(61.3 \%$ vs $56.3 \%, p=0.88 ; 58.3 \%$ vs $56.3 \%, p=0.991$ ). Non-relapse mortality and relapse incidence were $22.2 \%$ and $11.1 \%$, respectively. Severe acute-GvHD occurred in 4/63 patients. Transplant-related mortality was low at day+100 (17.5\%) and for the entire study period $(20.6 \%)$. Unexpectedly, few patients experienced mild-to-moderate toxicity, and main causes of death were infection and GvHD. BM blast counts, age, and donor-recipient gender-pairs did not affect the outcome. Less chemotherapy cycles prior to HSCT might result in more favorable outcome. Thus, haplo-HSCT with FA5-BUCY appears promising for advanced disease, especially when TBI and amsacrine, used for FLAMSA, are not available and in pediatric patients for whom TBI is not recommended.

\section{INTRODUCTION}

Despite significant advances in the treatment of leukemia, prognosis of patients with refractory or relapsed disease remains poor. Allogeneic hematopoietic stem cell transplantation (allo-HSCT) is now considered as the treatment with the highest probability of cure for primary refractory or relapsed leukemia [1]. As the availability of an HLA-matched sibling constitutes a barrier, especially in China because of the 1-child policy of the past 30 years, studies have shown that haploidentical HSCT (haploHSCT) with a graft from a partially matched family member is a possible alternative. In fact, a recent study in China by Huang et al. demonstrated that outcomes similar to those of identical-sibling transplant could be achieved with haplo-HSCT in patients with acute myeloid leukemia (AML) in first remission [2].

Preparative conditioning regimens prior to cell infusion are critical for reduction of tumor burden and immunoablation [3]. While earlier conditioning 
regimens used high-doses total body irradiation (TBI) and chemotherapeutic agents (myeloablative), new reducedintensity conditioning regimens have been proposed after the recognition that graft-versus-tumor effects contributed to the effectiveness of transplantation. Such protocol, i.e., the FLAMSA regimen, initially proposed by Schmid and colleagues [4] and consisting of sequential administration of aplasia-inducing chemotherapy (fludarabine, Ara-C, and amsacrine) followed by TBI plus cyclophosphamide (TBI/CY) or busulfan/CY (BU/CY), was found to be very effective for high-risk, refractory or relapsed AML [4-9]. However, this regimen could not be used at our center as amsacrine was not available in China, and we are not equipped to administer TBI.

As highly effective or standardized conditioning regimens have not been extensively tested for high-risk, relapsed/refractory leukemic patients, we applied the similar sequential treatment strategy as FLAMSA using a 5-day course of fludarabine and Ara-C for cytoreduction in the first phase, followed by BU/CY (named FA5-BUCY), and investigated the efficacy and safety of this regimen in patients with high-risk and advanced hematological malignancies. Here, we report the outcome of this cohort of 63 patients who were treated with haplo-HSCT after FA5-BUCY. The data show that FA5-BUCY is safe and can be successfully applied to relapsed/refractory patients who did not reach remission before transplantation.

\section{RESULTS}

Remission was achieved in 21 patients prior to the transplant, while 42 patients had no evidence of remission. Leukemic blasts in the bone marrow ranged from $7 \%$ 98\% (median: 35\%) in the patients who did not achieve hematological remission.

\section{Engraftment and donor chimerism}

All patients received fresh grafts containing a median of $7.9 \times 10^{8}$ mononuclear cells $/ \mathrm{kg}$ (range $4.2-21.4 \times 10^{8} / \mathrm{kg}$ ) and $5.33 \times 10^{6} \mathrm{CD} 34+$ cells $/ \mathrm{kg}$ (range $2.6-28 \times 10^{6} / \mathrm{kg}$ ) in total from $\mathrm{BM}$ and $\mathrm{PB}$ on day 0 . The time to neutrophil and platelet engraftment was 13 days (range 10-25) and 13 days (range 7-40), respectively. Two patients were not evaluable as one died before engraftment due to cerebral hemorrhage and the other died of severe bloodstream infection with both panresistant Pseudomonas Aeruginosa and Candida Tropicalis. For the other 61 patients, 95.1\% had complete donor chimerism at day 30 and thereafter, and $4.9 \%$ had mixed donor/recipient chimerism, which eventually converted to full donor chimerism during the 6 months follow-up.

\section{Toxicity}

Eighteen patients $(28.6 \%)$ experienced cytarabine syndrome (fever, maculopapular rash and conjunctivitis), which was easily controlled with corticosteroids. Gastrointestinal (GI) disturbances, which commonly include symptoms of anorexia, vomiting and diarrhea, occurred in some cases, but were very mild and did not generally need using total parenteral nutrition (TPN) support. Ten patients $(15.9 \%)$ developed toxic liver dysfunction, 7 with grade I hepatic toxicity, and 3 with grade II. Increase in bilirubin was observed, with a median value of $3.4 \mathrm{mg} / \mathrm{dl}$ (range, 2.1-6.2 mg/dl) and a median peak occurring on day 9 (range, days 6-15). All the patients who had hepatitis recovered fully and promptly with routine supportive treatment. None of the patients had severe organ failure.

\section{Overall outcome}

At the time of engraftment, 60 of the 61 patients (98.4\%) achieved complete remission, while one $(1.6 \%)$ had still not achieved remission even through engraftment was successful. At the time of analysis, $42 / 63$ patients $(66.7 \%)$ were still alive and in complete remission (CR), with a median follow-up time of 269 days (range:120-1081). Among these 42 patients, 30 patients $(71.4 \%)$ had not achieved remission before transplant. Seven patients $(11.1 \%)$ relapsed at median 133 days after transplant (range: 63-312).

Twenty-one patients died. The causes of death are summarized in Table 1. Fourteen patients (22.2\%) died from NRM, mainly from GvHD or infections. One patient $(1.6 \%)$ had secondary graft failure (GF) with uncontrolled cytomegalovirus (CMV) infection. Three patients died from cerebral hemorrhage $(n=1)$, suicide $(n=1)$ and electrolyte imbalance $(n=1)$. The 100-day mortality and TRM were $17.5 \%$ and $20.6 \%$ (excluding one case of suicide), respectively.

\section{GvHD}

The incidence of acute (a) and chronic (c) GvHD is summarized (shown in Supplementary Table S1). Eight patients (8/63) had grade I-II aGvHD. One patient had stage I skin GvHD, one had stage II gut GvHD, five had stage II aGvHD involving skin and liver, and one involving skin, liver and GI tract. Four patients (4/63) had grade III-IV aGvHD, either involving liver or GI tract. Four of these 12 patients died of complications related to severe aGvHD, and 1 died of relapse despite the presence of moderate aGvHD. All others who developed aGvHD are currently alive and disease-free. Six patients $(9.5 \%)$ had limited cGvHD. Among the patients with GVHD, 2 out of 10 patients who received pDLI had grade I-II aGvHD, one had limited cGvHD and one patient died of complications related to grade IV aGvHD involving the GI tract.

The major therapy administered for GvHD was systemic corticosteroids, whereas monoclonal antibodies 


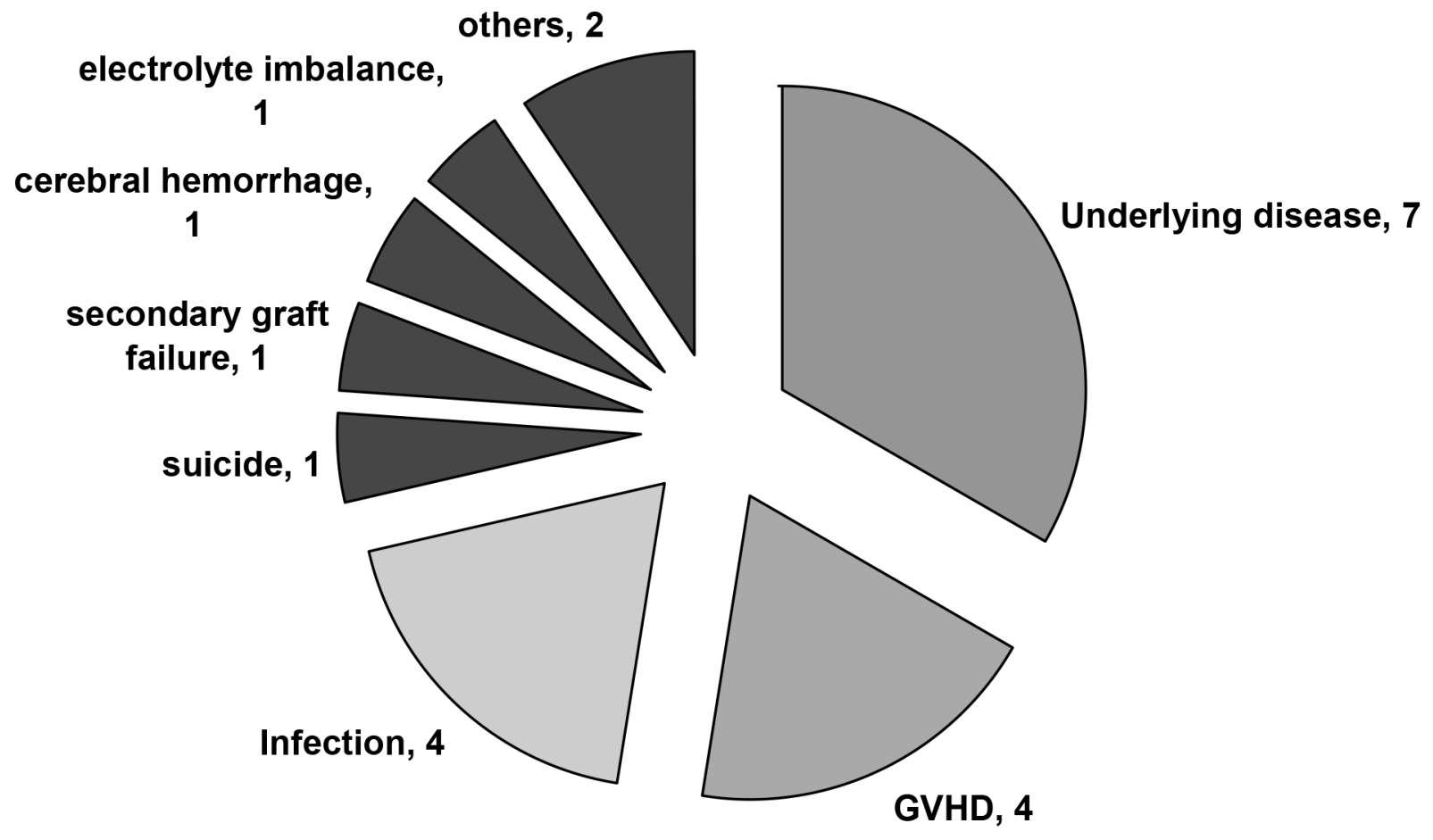

Twenty-one patients (21/63) died. Seven patients (7/21) died of relapse. Fourteen patients (14/21) died from NRM, mainly from GvHD or infections. One patient had secondary graft failure (GF) with uncontrolled cytomegalovirus (CMV) infection. Three patients died from cerebral hemorrhage $(n=1)$, suicide $(n=1)$ and electrolyte imbalance $(n=1)$.

(Basiliximab, Etanercept, and others) were used in 4 steroid-refractory cases.

\section{Disease control based on BM cellularity and minimal residual disease}

MRD assessment via WT1 expression and flow cytometry has been shown to correlate with disease status, and along with marrow blast counts, is a rapid and sensitive measure of treatment efficacy. Ten out of 42 patients who were not in remission were evaluated for disease response at different time points during HSCT (T I-IV; see Methods). They all exhibited disease prior to transplantation ( $\mathrm{T}$ I) with median marrow cellularity of $50 \%$ (range $10-90 \%$ ), overexpression of WT1 and positive MRD. However, after FA5BUCY conditioning (T III; Table 2), cytoreduction was successfully achieved in all 10 patients who demonstrated extreme hypocellularity of the bone marrow (range 0-30\%), reduced expression of WT1 below the cutoff value $(50 \%)[10,11,12]$, and negative $\operatorname{MRD}\left(<10^{-4}\right)[13,14]$, followed by a further decrease or stabilization after the engraftment (T IV). Only 1 patient (patient 4) showed a significant transient increase in BM blasts and WT1 expression, and a positive MRD after the 5-days of intensive chemotherapy with
FA; however, a further decrease was observed after administration of BUCY.

\section{Overall survival (OS) and relapse-free survival (RFS)}

The two-year OS and RFS in patients not in remission were similar when compared to those in complete remission $(61.3 \%$ vs $56.3 \%, p=0.88 ; 58.3 \%$ vs $56.3 \%, p=0.991$, respectively; Figure 1$)$. The outcome in the subgroup $\geq 14$-years $(\mathrm{n}=36$ ) was not significantly different than that in patients younger than 14 years of age $(\mathrm{n}=27)$ when taking into account all 63 patients $(2-$ year OS of $50.4 \%$ versus $70.5 \%, p=0.142$; Figure $2 \mathrm{~A}$ ) or only the subgroup not in remission (data not shown). Patients with a marrow blast count $\geq 20 \%(\mathrm{n}=8)$ prior to conditioning had a comparable outcome as those with marrow blasts $<20 \%(\mathrm{n}=34)$ (2-year OS $46.9 \%$ vs $59.1 \%, p=0.464$; Figure $2 \mathrm{~B}$ ). Patients who received $\leq 2$ cycles $(\mathrm{n}=17)$ of chemotherapy before haplo-HSCT seemed to have a better OS than those with $>2$ cycles $(\mathrm{n}=46)$ (2-year OS $77.8 \%$ vs $50.2 \%, p=0.094$; Figure $2 \mathrm{C})$; however, no significant difference was seen when comparing $\geq 5$ cycles $(n=22)$ and $<5$ cycles $(n=41)$ (Figure 2D). Contrary to what was previously reported [15], there was no significant impact of donor-recipient 
Table 2: BM cellularity and MRD assessment at different times during HSCT

\begin{tabular}{lccccccccccccc}
\hline $\begin{array}{l}\text { No. of } \\
\text { Cases }\end{array}$ & Diagnosis & \multicolumn{4}{c}{ Blast (\%) } & \multicolumn{4}{c}{ MRD/Flow } & \multicolumn{4}{c}{ WT1 (\%) } \\
\cline { 3 - 12 } & & T I & T II & T III & T IV & T I & T II & T III & T IV & T I & T II & T III & T IV \\
\hline 1 & AML & 36.5 & 14 & 7 & 0.5 & $4.4 \times 10^{-1}$ & $1.05 \times 10^{-1}$ & $<10^{-4}$ & $<10^{-4}$ & 88.51 & 56.38 & 12.88 & 38.02 \\
2 & AML & 12.5 & - & - & 1 & $1.24 \times 10^{-2}$ & $<10^{-4}$ & $<10^{-4}$ & $<10^{-4}$ & 93.11 & 50.77 & 2.5 & 0.37 \\
3 & AML & 8 & - & - & 0 & $4 \times 10^{-2}$ & $5 \times 10^{-3}$ & $<10^{-4}$ & $<10^{-4}$ & 57.39 & 16.50 & 8.84 & 0.08 \\
4 & ALL & 23.5 & 79 & 0.5 & 2 & $9 \times 10^{-2}$ & $3 \times 10^{-1}$ & $<10^{-4}$ & $<10^{-4}$ & 79.39 & 71.66 & 12.47 & 0.08 \\
5 & ALL & 39.5 & 23 & - & 1.5 & $2.8 \times 10^{-1}$ & $6 \times 10^{-2}$ & $<10^{-4}$ & $<10^{-4}$ & 97.01 & 50.3 & 23.6 & 0.07 \\
6 & SAML(MDS) & 98 & 40.5 & 15.5 & 2.5 & $1 \times 10^{-1}$ & $8 \times 10^{-3}$ & $<10^{-4}$ & $<10^{-4}$ & 95.06 & 84.05 & 27.37 & 3.41 \\
7 & AML & 95 & 40 & 18 & 2 & $8.2 \times 10^{-1}$ & $4.88 \times 10^{-1}$ & $3.5 \times 10^{-2}$ & $<10^{-4}$ & 95.68 & 83.5 & 72.01 & 42.06 \\
8 & SAML(MDS) & 11 & 8.5 & - & 1.5 & $3.9 \times 10^{-1}$ & $4.3 \times 10^{-1}$ & $<10^{-4}$ & $<10^{-4}$ & 65.2 & 46.44 & 30.9 & 11.70 \\
9 & AML & 45 & - & - & 0.5 & $4.43 \times 10^{-1}$ & $<10^{-4}$ & $<10^{-4}$ & $<10^{-4}$ & 84.32 & 21 & 11.5 & 0.52 \\
10 & AML & 88 & 10 & - & 0.5 & $54.91 \times 10^{-1}$ & $3.9 \times 10^{-1}$ & $<10^{-4}$ & $<10^{-4}$ & 74.44 & 30.9 & 11.7 & 0.15 \\
\hline
\end{tabular}

Ten evaluable patients exhibited disease at the time of transplantation as shown by WT1 overexpression and positive MRD. The disease responses were measured at different time points defined as: time point I (T I), before the initiation of administration of fludarabine and cytarabine; time point II (T II), right after completion of the 5-days FA; time point III (T III), right after conditioning with BUCY; and time point IV (T IV), at the time of hematological engraftment. A decrease in the number of marrow blasts $(<5 \%)$, downexpression of WT1 below the cutoff value $(50 \%)$, and negative $\operatorname{MRD}\left(<10^{-4}\right)$ were documented after the FA5-BUCY conditioning, followed by a further decrease or stabilization after the engraftment. Only 1 patient (patient 4) showed a significant transient increase of marrow blasts, WT1 overexpression and positive MRD after the 5-days intensive chemotherapy of FA, then a further decrease after the BUCY regimen.

gender pairs on OS in our study (Figure 2E). Although the numbers were too small for statistical studies, GvHD did not seem to affect outcome (data not shown). Thirty-two out of the 42 patients not in remission did not receive DLI post-HSCT due to the presence of active infection, aGvHD and other disorders; however, there was no significant difference in the 2-yr RFS when compared to patients who receive DLI (Supplementary Figure S1).

\section{Comparison with historical control}

Conditioning regimens such as BUCY or CYTBI are considered as the conventional conditioning regimens for allo-HSCT in patients with leukemia or MDS. Tang et al. compared their study of allo-HSCT in AML patients to a historical control series composed of 26 patients who received allo-HSCT between January 2000 and May 2011 after conventional conditioning regimens [9]. To demonstrate the effectiveness of FA5BUCY in the 42 patients not in remission from our study group, we similarly compared the outcome of our patients to that of this previously reported historical control series [9]. Patient characteristics, GvHD prophylaxis and graft sources were comparable in both groups (Table 3). Although the tumor burden evaluated by marrow blasts was much higher in our study group, preparation with the FA5-BUCY regimen resulted in significantly improved 2 -year relapse rates $(11.9 \%$ vs $81.2 \%, \mathrm{p}<0.001)$, NRM $(26.2 \%$ vs $40.9 \%, \mathrm{p}<0.001)$, RFS $(58.3 \%$ vs $11.11 \%, \mathrm{p}<0.001)$ and OS $(61.3 \%$ vs $11.11 \%, \mathrm{p}<0.001)$.

\section{DISCUSSION}

Complete remission is rarely achieved in patients with relapsed or refractory acute leukemia despite the development of new investigational therapies. Allo-HSCT is currently the treatment with the highest probability of cure, allowing the effective elimination of the leukemiainitiating cells. However, the disease status prior to HSCT, often uncontrolled, has limited the overall survival, even among younger patients with a well-matched donor [16-18]. With conventional conditioning regimens such as BUCY or CY-TBI, most patients who were not in remission at the time of HSCT relapsed rapidly within 6 months of transplantation, as previously reported [9] and as we have experienced (personal communication). Nevertheless, sequential treatment strategies including intensive chemotherapy to reduce disease burden followed by reduced-intensity conditioning have been prospectively shown to improve treatment outcome in leukemias, especially for high-risk, relapsed/refractory patients with 
active disease [4-9, 19]. Among those, the FLASMAbased protocol (fludarabine/amsacrine/cytarabine) has been extensively used with great success. Furthermore, a novel active agent, clofarabine, which has no overlapping toxicities to the conditioning regimen, has been used as a pre-conditioning, intensification cytoreduction agent [19].
Here, we evaluate the efficacy and safety profile of the FA5-BUCY regimen in 63 acute leukemic patients either at high risk $(\mathrm{n}=21)$ or not in remission (median marrow blasts of $35 \%$ pre-HSCT, $n=42$ ). All these patients could not receive HLA identical allo-grafts due to the lack of HLA identical siblings.

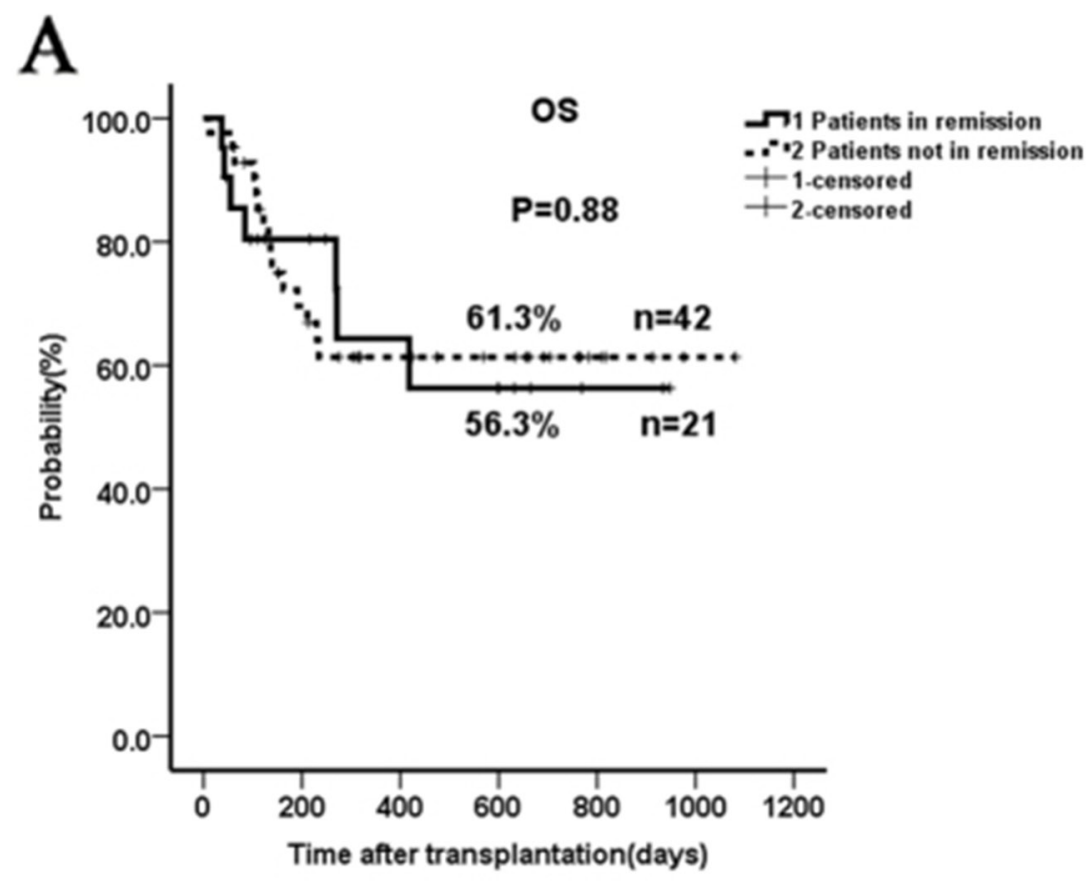

B

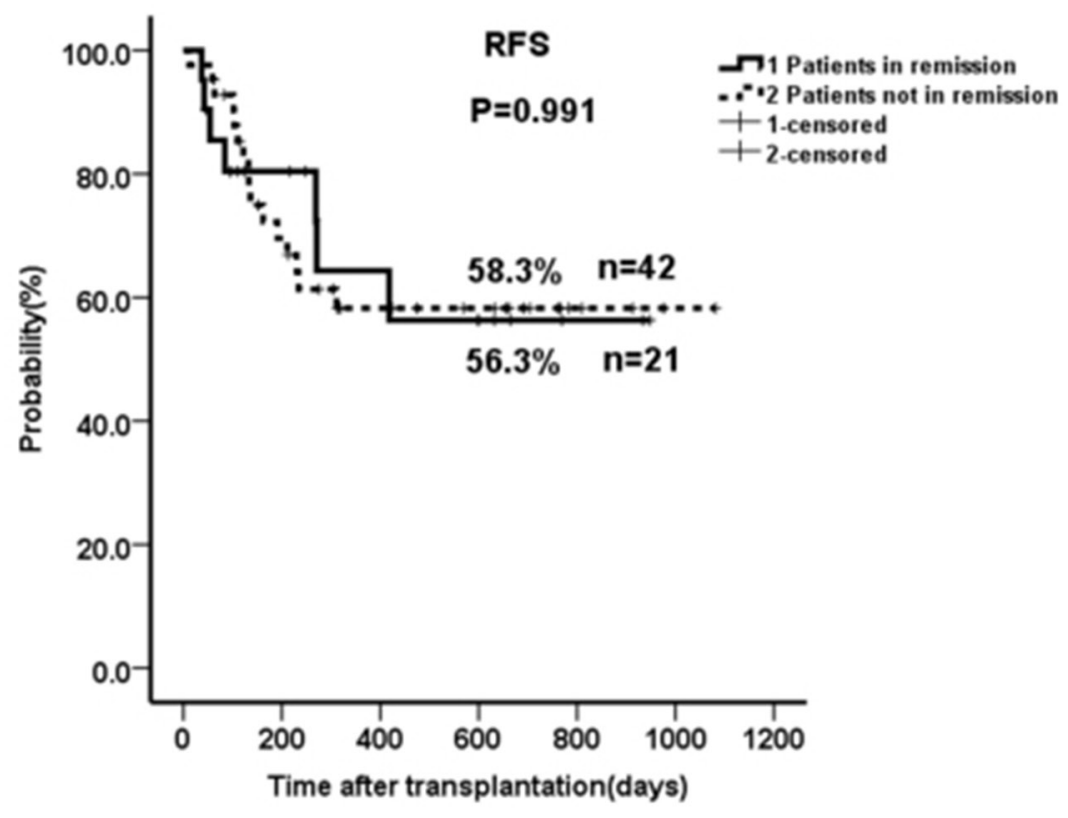

Figure 1: Two-year OS and RFS in patients not in remission prior to HSCT compared to those in complete remission. The two-year OS and RFS in patients not in remission were similar to those from patients in complete remission $(61.3 \% \mathrm{vs} 56.3 \%, p=0.88$; $58.3 \%$ vs $56.3 \%, p=0.991)$. 
A

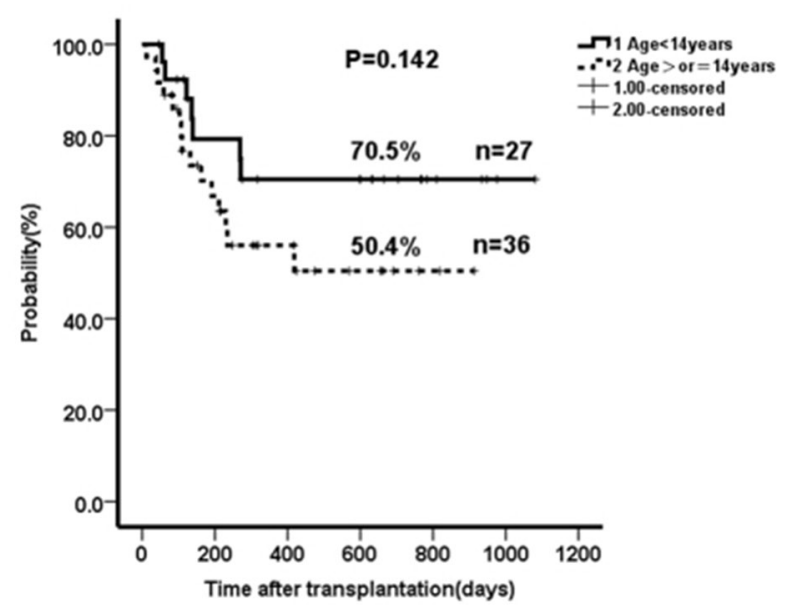

B

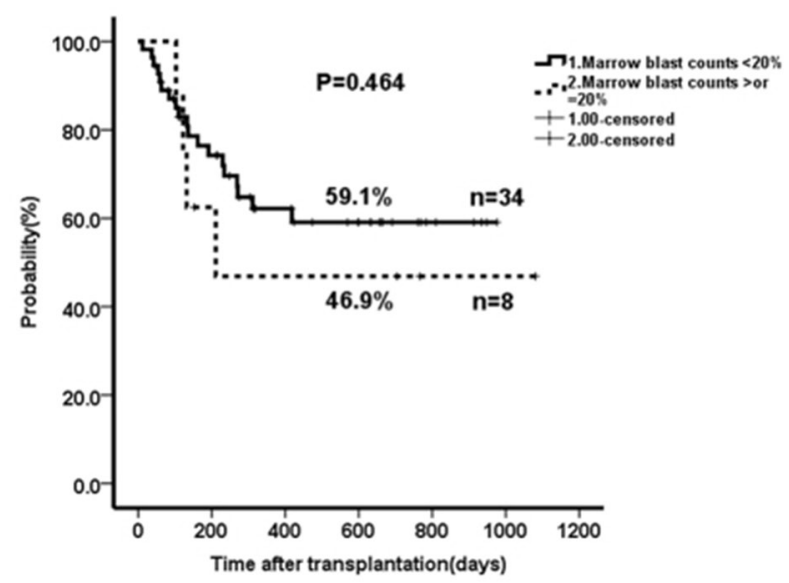

C

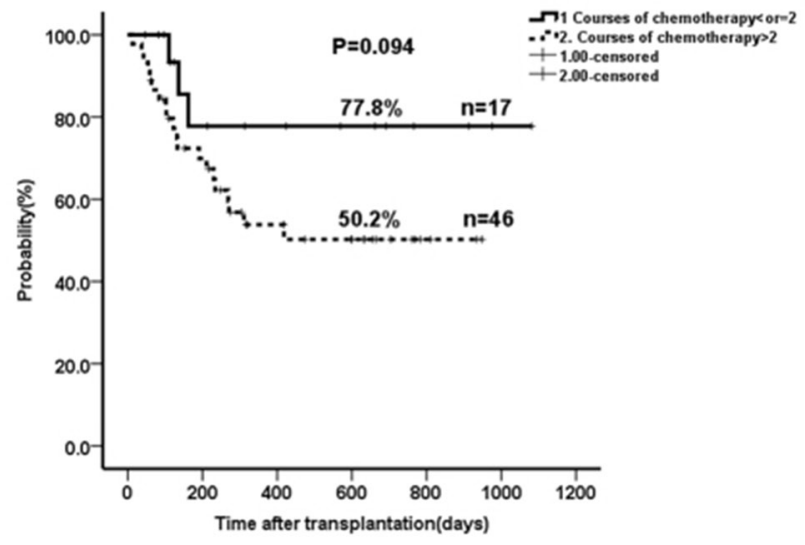

Figure 2: The impact of age, marrow blasts, previous cycles of chemotherapy and donor-recipient gender pairs on overall survival. A. Two-year OS in patients with age $\geq 14$ years compared to those with $<14$ years. B. Two-year OS in patients with marrow blast count $\geq 20 \%$ compared to those with $<20 \%$. C. Two-year OS in patients who received $\leq 2$ cycles of chemotherapy prior-HSCT compared to those with $>2$ cycles. (Continued) 


\section{$\mathrm{D}$}

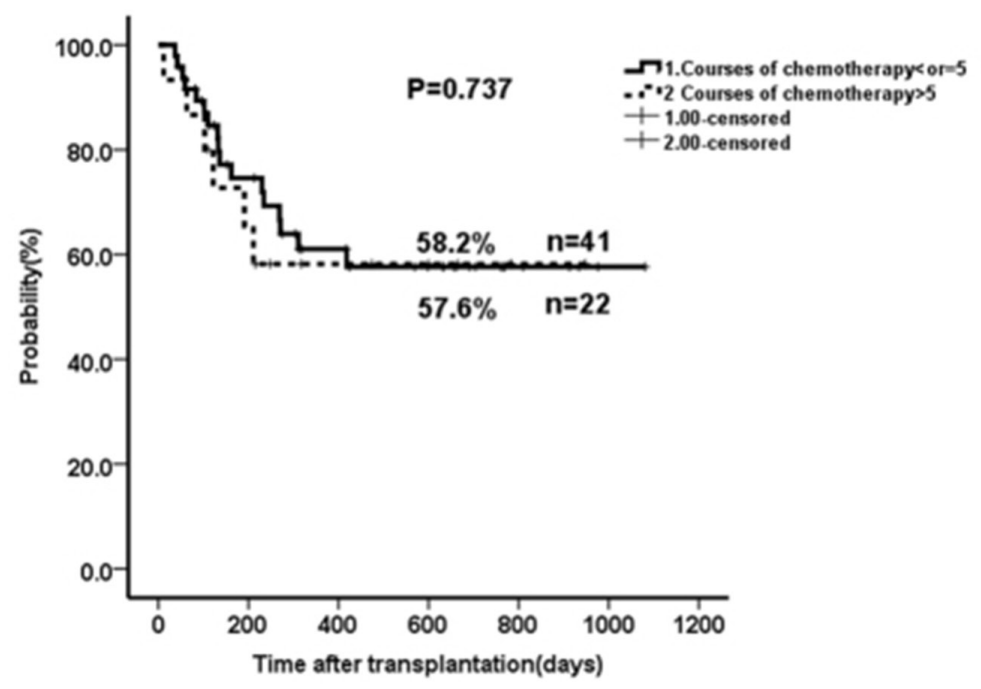

$\mathrm{E}$

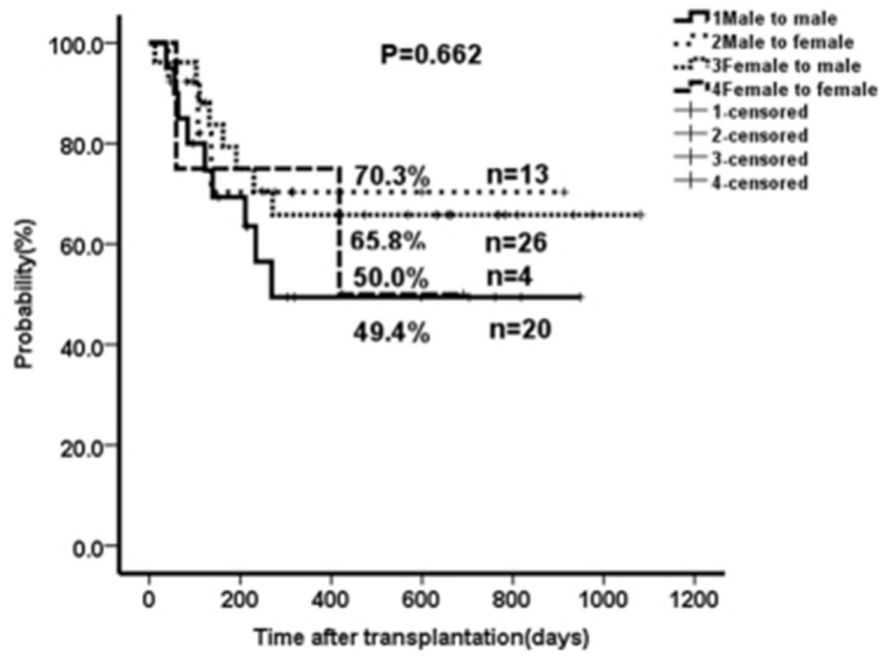

Figure 2: (Continued)The impact of age, marrow blasts, previous cycles of chemotherapy and donor-recipient gender pairs on overall survival. D. Two-year OS in patients who received $>5$ cycles of chemotherapy prior-HSCT compared to those with $\leq$ 5 cycles. E. Two-year OS in patients who received different donor-recipient gender pairs. The outcome in the subgroup $\geq 14$ years $(n=36)$ was not significantly different than that in patients younger than 14 years of age $(n=27)$ when taking into account all 63 patients (2-year OS of $50.4 \%$ versus $70.5 \%, \mathrm{p}=0.142$; Figure $2 \mathrm{~A}$ ). Patients with a marrow blast count $\geq 20 \%(\mathrm{n}=8)$ prior to conditioning had a comparable outcome as those with marrow blasts $<20 \%$ ( $n=34)$ ( 2 -year OS $46.9 \%$ vs $59.1 \%, \mathrm{p}=0.464$; Figure $2 \mathrm{~B}$ ). Patients who received $\leq 2$ cycles $(\mathrm{n}=17)$ of chemotherapy before haplo-HSCT seemed to have a better OS than those with $>2$ cycles $(\mathrm{n}=46)$ ( 2 -year OS $77.8 \%$ vs $50.2 \%$, $\mathrm{p}=0.094$; Figure 2C); however, no significant difference was seen when comparing $>5$ cycles $(\mathrm{n}=22)$ and $\leq 5$ cycles $(\mathrm{n}=41)$ (Figure 2D). There was no significant impact of donor-recipient gender pairs on OS (Figure 2E).

The combination of fludarabine and Ara-C/ cytarabine in a FLAG-based regimen has been used as the salvage treatment for patients with relapsed/refractory leukemia and is well-tolerated, although as a secondline chemotherapy regimen, it has limited efficacy on achieving remission for relapsed/refractory patients [20, 21]. Our analysis demonstrated that the Regimen of a 5-day course of FA combined with Bucy still resulted in significant cytoreduction prior to HSCT, as evidenced by a decrease in BM blasts and MRD, It was previously reported that for each $10 \%$ increase in marrow blasts at the time of HSCT, there was an increased risk of death by a factor of 1.21 [22]. Thus, the early removal of blasts by the combination of FA with Bucy before stem cell infusion may offer benefits, resulting in transplant tolerance and better survival $[23,24]$ Our study enrolled 42 patients 
Table 3: Characteristics and transplant outcome of patients not in remission in our study group and historical group ${ }^{9}$

\begin{tabular}{|c|c|c|c|}
\hline & $\begin{array}{l}\text { Study group } \\
\text { (42 cases) }\end{array}$ & $\begin{array}{l}\text { Historical group } \\
\text { (26 cases) }\end{array}$ & P value \\
\hline Age, median (range) & $23(1.1-49)$ & $35(18-5)$ & \\
\hline \multicolumn{4}{|l|}{ Sex } \\
\hline Male & 31 & 15 & 0.197 \\
\hline Female & 11 & 11 & \\
\hline Marrow blasts (\%) & & & 0.002 \\
\hline Blasts $<5 \%$ & 0 & 6 & \\
\hline Blasts $\geq 5 \%$ & 42 & 20 & \\
\hline \multicolumn{4}{|l|}{ Graft sources } \\
\hline BM & 0 & 1 & \\
\hline PBSC & 0 & 25 & \\
\hline $\mathrm{BM}+\mathrm{PBSC}$ & 42 & & \\
\hline \multicolumn{4}{|l|}{ GvHD prophylaxis } \\
\hline $\mathrm{ATG}+\mathrm{CSA}+\mathrm{MMF}+\mathrm{MTX}$ & $42(100 \%)$ & $26(100 \%)$ & \\
\hline Transplant outcome (2-years) & & & $<0.001$ \\
\hline Relapse rate & $11.9 \%$ & $81.2 \%$ & \\
\hline Non relapse mortality & $26.2 \%$ & $40.9 \%$ & \\
\hline Relapse free survival & $58.3 \%$ & $11.11 \%$ & \\
\hline Overall survival & $61.3 \%$ & $11.11 \%$ & \\
\hline
\end{tabular}

with molecular or hematological (7-98\% BM blasts) advance disease prior to HSCT, who had relapsed or were refractory to the standard frontline treatment, and did not respond to multiple cycles of salvage treatments. These patients are usually not considered as good candidates for transplantation, however, our results revealed that HSCT with FA5-BUCY conditioning brought about a rapid MRD remission and efficiently reduced tumor burden before stem cell infusion. Moreover, $98.4 \%$ did achieve complete remission at the time of engraftment, and the reduction of disease burden had a favorable impact on outcome. The 2-year OS (61.3\%) and RFS (58.3\%) were similar in patients not in remission before HSCT compared to patients in remission. There may be a statistical bias because of the lower number of patients in the remission group, nevertheless, the OS were better than those reported in previous studies, which is very encouraging for treating these patients $[4-9,19]$. Although haploidentical transplantation might be expected to be associated with lower relapse rate and disease-free survival because of a more potent graft-versus-leukemia (GVL) effect, and no difference in relapse rate was found whether or not DLI was used, we believe that decreasing tumor burden with FA5-BUCY is the key factor to improve outcome, especially when compared to the outcomes following conventional conditioning regimens. Importantly, these favorable outcomes were not affected by the blast counts in the bone marrow, the age of the patients or the presence of GvHD, indicating that haplo-HSCT with FA5BUCY regimen is beneficial to the high-risk, relapsed/ refractory leukemic patients regardless of the percentage of bone marrow blasts at the time of HSCT. In addition, it is believed that children have a much better prognosis than adults because children usually do not have the comorbidities typically found in adults (e.g., chronic hypertension, diabetes mellitus, chronic coronary ischemic disease, smoking-related illnesses). Our results indicate that FA5-BUCY regimen can be used in pediatric or adult patients, especially in pediatric patients for whom TBI is not recommended.

As chemotherapy can also affect the rapidly growing and dividing normal cells, such as those in the bone marrow, digestive tract, skin, hair and reproductive organs, less chemotherapy may be helpful to minimize the risk of infection, graft failure and GvHD, and such protocols merit further investigation. Our data reveal that less cycles of chemotherapy prior to HSCT may lead to a more favorable clinical outcome. Thus, it suggests that 
transplant decision should be made as early as possible once the patients start developing resistance to the chemotherapy. For patients who do not have a matched sibling donor, our data show that a haploidentical family donor can be an alternative option. As a parent is always a haploidentical match for the children and vice versa, it is reasonable to test the close family members early to proceed quickly with this life-saving transplant and avoid the waiting periods often occurring with unrelated donors. Interestingly, our analysis did not find any negative impact of patient-donor gender pairs on transplant outcome (Figure 2E), contrary to previous reports $[15,25,26]$. It indicates that the donor source is not a major limitation, making haplo-HSCT a more feasible alternative.

While FA5-BUCY is an intensive regimen, it was unexpectedly associated with only mild to moderate toxicity. According to the assessed EBMT risk score, most of the patients enrolled in the study were fit and suitable for allo-HSCT as recommended [1]. One of the most important findings in the present analysis is that the probability of TRM in such a high-risk cohort was low, both at day +100 $(17.5 \%)$ and for the entire study period $(20.6 \%)$, and that the majority of deaths was attributed to early transplantrelated complications, which may be associated with active disease. The major side effects of FA5-BUCY were gastrointestinal and hepatic toxicity, which can be resolved within a relatively short time after prompt and appropriate management. The fact that only rare cases of severe mucositis, a common complication post-HSCT related to high-intensity conditioning $[9,27,28]$ were observed, resulted in better quality of life, treatment adherence, and ultimately improved outcome in these patients.

Taken together, our data demonstrate that FA5BUCY as an intensive conditioning regimen prior to haplo-HSCT presents advantages for leukemic patients who did not reach remission, and does not increase NRM. While we didn't compare haplo to matched donors in our current study, we suggest that haploSCT can be an alternative option, including with the mother as the donor, especially when the refractory patients urgently need to undergo this salvage therapy. In addition, haplo-SCT can be performed rapidly because the donors are all close family members. As the heterogeneity of the data and the size of the population may have been limiting factors, further investigations with larger cohorts are warranted.

\section{MATERIALS AND METHODS}

\section{Study design}

We conducted a nonrandomized, observational study from a single center in a cohort of high-risk, primary refractory or relapsed patients with hematological malignancies who underwent conditioning with the FA5-
BUCY regimen, registered on http://ClinicalTrials.gov (NCT02328950), and haplo-HSCT at the Union Hospital, Fujian Medical University, Fujian Province, China between Feb. 2013 and Dec. 2015. Informed consent was obtained from all patients and donors before being included in the study. This study was conducted in accordance with the ethical standards of the local institutional review board and with the Helsinki Declaration.

\section{Patient characteristics}

Patient characteristics are summarized in Table 4. Sixty-three consecutive patients (19 females, 44 males) with a median age of 20 years (range 1.1-49) were included in the study. 42 patients had relapsed $(n=34)$ or primary refractory $(n=8)$ leukemia, and 21 patients had leukemia in remission but had a high risk of relapse per cytogeneics and/ or molecular markers based on the National Comprehensive Cancer Network practice guidelines [29]. All patients had received standard frontline treatment. After failure of more than 2 cycles of salvage treatments, $15 / 42$ patients had relapsed/refractory diseases without molecular remission, and 27/42 had hematological disease with 7\%-98\% BM blasts (median 35\%) prior to HSCT".

Primary refractory AML was defined as blast persistence in bone marrow (BM) aspirates on day 28 after the first or second induction treatment. Relapsed AML was defined as $>5 \%$ blasts in BM aspirates in patients who achieved complete cytological remission after the first or second induction treatment. The diagnoses were as follows: acute myeloid leukemia (AML, n=29), AML secondary to myelodysplastic syndrome ( $\mathrm{AML}, \mathrm{n}=8$ ), acute lymphoblastic leukemia (ALL, n=19), advanced myelodysplastic syndrome $(\mathrm{aMDS}, \mathrm{n}=5)$ and chronic myelogenous leukemia in blast crisis (CML-BC, $n=2)$.

\section{HLA typing and donors}

Patients and their donors were tested for HLA-A, HLA-B, HLA-C, HLA-DRB1, and HLA-DQB1 by highresolution molecular typing methods. All donors were family members who shared one HLA haplotype with the recipient, but differed to various degrees for the HLA-A, B, and DR antigens of the unshared HLA haplotype. Parents accounted for $57.1 \%$ of the donor sources.

All patients received both BM and peripheral blood (PB) mobilized with granulocyte colony-stimulating factor (G-CSF; $5 \mu \mathrm{g} / \mathrm{kg}$ of body weight per day for 5 days), as described by Huang et al [2]. The target mononuclear cell counts in total from BM and PB were $>4 \times 10^{8} / \mathrm{kg}$ of recipient weight.

\section{EBMT risk score}

To assess the risks of haplo-HSCT, the European Group for Blood and Marrow Transplantation (EBMT) risk 


\section{Table 4: Patient Characteristics}

Median age, years (range)

Sex

Male

Female

Primary disease

AML

MDS-SAML

ALL

Advanced MDS

CML-BC

Remission status

In remission

Not in remission

Donor-recipient relationship

Parents-Children

Siblings

Children-Parents

Donor-recipient blood type

Compatibility

Major ABO incompatibility

Minor ABO incompatibility

Major and minor ABO incompatibility

EBMT risk scores

0-2

3-4

5-7

Donor-recipient gender pairs

$\mathrm{M}$ to $\mathrm{M}$

$\mathrm{M}$ to $\mathrm{F}$

F to $\mathrm{M}$

$\mathrm{F}$ to $\mathrm{F}$

Engraftment

Neutrophils, day (range)

Thrombocytes, day (range)

Infusion dose CD34+, 106/kg (range)

Follow-up time, days (range)
$20(1.1-49)$

$44(69.8 \%)$

$19(30.2 \%)$

$29(46.0 \%)$

$8(12.7 \%)$

$19(30.2 \%)$

$5(7.9 \%)$

$2(3.2 \%)$

$21(33.3 \%)$

$42(66.7 \%)$

$36(57.1 \%)$

$21(33.3 \%)$

$6(9.5 \%)$

$34(54.0 \%)$

$11(17.5 \%)$

$15(23.8 \%)$

$3(4.8 \%)$

$36(57.1 \%)$

$23(36.5 \%)$

$4(6.3 \%)$

$20(31.7 \%)$

$13(20.6 \%)$

$26(41.3 \%)$

$4(6.3 \%)$

$13(10-25)$

$13(7-40)$

$5.33(2.6-28)$

$269(120-1081)$ 
score [30] was calculated for each individual patient before transplantation based on 5 pre-transplantation variables: age, disease stage, time from diagnosis to transplantation, donor type, and donor-recipient sex combination.

\section{Conditioning regimens, transplantation and GvHD prophylaxis}

The FA5-BUCY protocol is shown in Table 5. All 63 patients received the aplasia-inducing salvage therapy consisting of $30 \mathrm{mg} / \mathrm{m}^{2} /$ day Fludarabine and high-dose $2 \mathrm{~g} / \mathrm{m}^{2} /$ day Ara-C (Cytarabine) for 5 consecutive days from day -13 to day -9 , followed after 1 day of rest by $3.2 \mathrm{mg} / \mathrm{kg} /$ day BU from day -7 to day -5 and $1.8 \mathrm{~g} / \mathrm{m}^{2} /$ day CY from day -4 to day -3 .

Prophylactic donor lymphocyte infusions (DLI) were given from day +30 in patients without evidence of graft versus host disease (GvHD) or severe infection, as recommended by Kolb et al [31]. Peripheral blood mononuclear cells from donors were cryopreserved and adjusted to a defined CD3 $+\mathrm{T}$ lymphocyte count $\left(5 \times 10^{5}\right.$ to $1 \times 10^{6} \mathrm{CD} 3+$ cells $/ \mathrm{kg}$ ) before transplantation.

GvHD prophylaxis consisted of rabbit antithymocyte globulin ( 20 patients with Thymoglobulin, Genzyme, $10 \mathrm{mg} / \mathrm{kg}$ and 23 with ATG-Fresenius ${ }^{\circledR}, 40$ $\mathrm{mg} / \mathrm{kg}$ ) from day -4 to day-1, cyclosporine A (plasma level 100-250 ng/ml, starting from day -10 , and tapered from the second or third month if no signs of GvHD were present), mycophenolate mofetil ( $5 \mathrm{mg} / \mathrm{kg}$ bid, starting from day +7 and tapered after engraftment), and shortterm methotrexate (MTX, $15 \mathrm{mg} / \mathrm{m}^{2}$ at day +1 , and $10 \mathrm{mg}$ / $\mathrm{m}^{2}$ at day +3 and +6$)$.
Acute GvHD was graded according to the Glucksberg criteria [32]. Chronic GvHD was graded according to the revised Seattle classification [33].

All patients were treated according to our institutional transplant guidelines for antiviral, antifungal, and antimicrobial prophylaxis.

\section{Toxicity}

Regimen-related toxicity was graded according to the scale of the NCI Common Terminology Criteria for Adverse Events (CTCAE) version 4.0 [34, 35].

\section{Engraftment}

Engraftment was assessed by peripheral blood counts. Neutrophil engraftment was defined as the first of two consecutive days with an absolute neutrophil count over 500 neutrophils/L. Platelet engraftment was defined as the first of two consecutive days with more than 20,000 platelets/L without platelet transfusion.

The hematopoietic donor cell chimerism status of mononuclear cells and CD3 $+\mathrm{T}$ cells was monitored in all patients using microsatellite markers, as described [36].

\section{Minimal residual disease}

To evaluate the cytoreduction effects and disease response of FA5-BUCY regimen, a BM aspirate and a biopsy were performed to evaluate the kinetics of the BM blasts from 10 evaluable patients at different time points

Table 5: Protocol for FA5-BUCY conditioning regimen

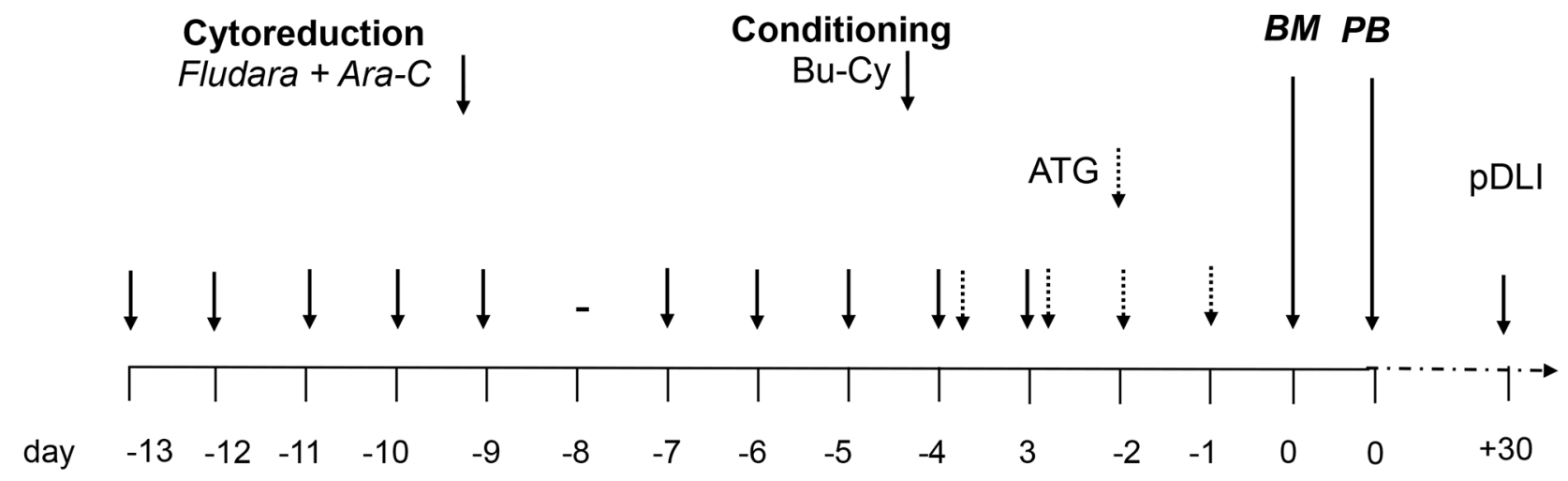

CSA

Immunosuppression:

MMF

The FA5-BUCY regimen consists of $30 \mathrm{mg} / \mathrm{m}^{2} /$ day Fludarabine and high-dose $2 \mathrm{~g} / \mathrm{m}^{2} /$ day Ara-C (Cytarabine) for $5 \mathrm{consecutive}$ days from day -13 to day -9 , followed after 1 day of rest by $3.2 \mathrm{mg} / \mathrm{kg} /$ day BU from day -7 to day -5 and $1.8 \mathrm{~g} / \mathrm{m}^{2} / \mathrm{day} \mathrm{CY}$ from day -4 to day -3 . All patients received stem cells from both bone marrow (BM) and peripheral blood (PB). GvHD prophylaxis included ATG, CsA, MMF and short-term MTX. 
defined as: time point I ( $\mathrm{T} \mathrm{I}$ ), before the initiation of administration of fludarabine and cytarabine; time point II (T II), right after completion of the 5-days FA; time point III (T III), right after conditioning with BUCY; and time point IV (T IV), at the time of hematological engraftment. At each time point, the Wilms tumor gene 1 (WT1) dynamic expression was also assessed using quantitative RT-PCR $[10,11]$, and minimal residual disease (MRD) was detected by multiparametric flow cytometry (FC) with a Cytomics FC-500 flow cytometer (Beckman-Coulter, Brea, CA) and the following combinations of monoclonal antibodies (mAbs): FITC, PE, PE-Texas Red, and PE-Cy5: CD13/ CD33/CD45/CD34, CD38/CD56/CD45/CD34, CD15/ CD14/CD45/CD34, CD15/CD13/CD34/CD33, CD64/ CD11B/CD45/CD34, HLA-DR/ CD117/CD34/CD33, CD5/ CD117/CD34/CD33, CD7/CD34/CD45/CD2, CD20/CD19/ CD34/CD45, CD41/CD235a/CD45/CD34, and CD45/CD4/ $\mathrm{CD} 8 / \mathrm{CD} 3$. A percentage of leukemic cells exceeding $10^{-4}$ was considered as evidence of $\operatorname{MRD}[13,14]$.

\section{Statistics}

Data were analyzed with the SPSS 13 statistical package (Chicago, IL, USA). Overall survival (OS) and relapse-free survival (RFS) were calculated using the Kaplan-Meier (KM) estimates. OS was defined as the time from HSCT to death from any cause, and RFS was defined as the time from HSCT to relapse. The log-rank test was used for the comparison of KM estimates between different groups of patients with a significance level of 0.05 . Proportional hazards were calculated with the semiparametric Cox regression model. Relapse was defined as recurrence of BM blasts $>5 \%$, reappearance of blasts in the blood, or development of extramedullary disease infiltrates at any site, Non-relapse mortality (NRM) defined as death without evidence of disease recurrence post-HSCT.

\section{ACKNOWLEDGMENTS}

This work was supported in part by the National and Fujian Provincial Key Clinical Specialty Discipline Construction Program, China, National High Technology Research and Development Program of China, 863 program (2012AA02A505), National Public Health Grand Research Foundation (201202017), National Natural Science Foundation of China (81570162), Fujian Provincial Key Laboratory Foundation of Hematology (2009J1004). We thank Prof. Jiong $\mathrm{Hu}$ for providing us with the clinical data for the historical control series. We also thank Prof. Hans-Jochem Kolb for the stimulating discussions and his encouragement when the study was initiated. The authors would like to thank the patients, their parents and all the nurses and physicians at the Center who all contributed to the management of patient care, and Dr. Martine Torres for her critical reading of the manuscript and editorial assistance.

\section{CONFLICTS OF INTEREST}

The authors declare no competing financial interests.

\section{Authorship contributions}

Prof. Jianda $\mathrm{Hu}$ and Prof. Ting Yang designed and performed the research. Dr. Jinhua Ren and Dr. Qiaoxian Lin collected the data. Dr. Qiaoxian Lin performed the statistical analyses. Prof. Ting Yang interpreted the data and wrote the manuscript. All authors participated in the management of patient care. All authors critically reviewed and approved the manuscript.

\section{Key points}

- The 5-day Fludarabine/Ara-C cytoreductive chemotherapy, which resulted in better disease control prior to HSCT, can safely be used to treat refractory/ relapsed leukemic patients before transplantation when TBI or amsacrine are not available.

- Haploidentical transplantation is a promising alternative for advanced hematological malignancies in pediatric and adult patients, especially when this lifesaving transplant is urgently needed.

\section{REFERENCES}

1. Thol F, Schlenk RF, Heuser M, Ganser A. How I treat refractory and early relapsed acute myeloid leukemia. Blood. 2015;126:319-327.

2. Wang Y, Liu QF, Xu LP, Liu KY, Zhang XH, Ma X, Fan ZP, $\mathrm{Wu} \mathrm{DP}$, Huang XJ. et al. Haploidentical vs identical-sibling transplant for AML in remission: a multicenter, prospective study. Blood. 2015;125:3956-3962.

3. Gyurkocza B, Sandmaier BM. Conditioning regimens for hematopoietic cell transplantation: one size does not fit all. Blood. 2014;124:344-353.

4. Schmid C, Schleuning M, Schwerdtfeger R, Hertenstein B, Mischak-Weissinger E, Bunjes D, Harsdorf SV, Scheid C, Holtick U, Greinix H, Keil F, Schneider B, Sandherr $\mathrm{M}$, et al. Long-term survival in refractory acute myeloid leukemia after sequential treatment with chemotherapy and reduced-intensity conditioning for allogeneic stem cell transplantation. Blood. 2006;108:1092-1099.

5. Detrait M, Chevallier P, Sobh M, Guillaume T, Thomas $\mathrm{X}$, Morisset S, Tedone N, Delaunay J, Nicolini F, Ducastelle S, Chelghoum Y, Barraco F, Labussiere H, et al. Outcome of High-risk and refractory AML/MDS patients receiving a Flamsa sequential chemotherapy regimen followed by reduced-Intensity conditioning (RIC) and allogeneic hematopoeitic stem cell transplantation (alloHSCT). Biology of Blood and Marrow Transplantation. 2012;18:S335-S336. 
6. Hemmati P, Terwey T, Vuong L, Na K, Coutre PL, Dörken $\mathrm{B}$, Arnold R. Long-term outcome of allogeneic stem cell transplantation for acute myeloid leukemia beyond first complete remission-a single center experience. Biology of Blood and Marrow Transplant. 2014;20:S154.

7. Krejci M, Doubek M, Dusek J, Brychtova Y, Racil Z, Navratil M, Tomiska M, Horky O, Pospisilova S, Mayer J. Combination of fludarabine, amsacrine, and cytarabine followed by reduced-intensity conditioning and allogeneic hematopoietic stem cell transplantation in patients with high-risk acute myeloid leukemia. Annals of hematology. 2013;92:1397-1403.

8. Schneidawind D, Federmann B, Faul C, Vogel W, Kanz L, Bethge WA. Allogeneic hematopoietic cell transplantation with reduced-intensity conditioning following FLAMSA for primary refractory or relapsed acute myeloid leukemia. Annals of hematology. 2013;92:1389-1395.

9. Tang W, Fan X, Wang L, Hu J. Busulfan and fludarabine conditioning regimen given at hematological nadir of cytoreduction fludarabine, cytarabine, and idarubicin chemotherapy in patients with refractory acute myeloid leukemia undergoing allogeneic stem cell transplantation: a single arm pilot consort study. Medicine. 2015;94:e706.

10. Borowitz MJ, Devidas M, Hunger SP, Bowman WP, Carroll AJ, Carroll WL, Linda S, Martin PL, Pullen DJ, Viswanatha D, Willman CL, Winick N, Camitta BM; Children's Oncology Group. Clinical significance of minimal residual disease in childhood acute lymphoblastic leukemia and its relationship to other prognostic factors. A Children's Oncology Group study. Blood. 2008;111:5477-5485.

11. won M, Martínez-Laperche C, Infante M, Carretero F, Balsalobre P, Serrano D, Gayoso J, Pérez-Corral A, Anguita J, Díez-Martín JL, Buño I. Evaluation of minimal residual disease by real-time quantitative PCR of Wilms' tumor 1 expression in patients with acute myelogenous leukemia after allogeneic stem cell transplantation: correlation with flow cytometry and chimerism. Biol Blood Marrow Transplant. 2012;18:1235-1242.

12. Willasch AM, Gruhn B, Coliva T, Kalinova M, Schneider G, Kreyenberg H, Steinbach D, Weber G, Hollink IHIM, Zwaan CM, Biondi A, der Velden VHJ van, D Reinhardt, et al. on behalf of the European Study Group on WT1 Expression in Childhood AML. Standardization of WT1 mRNA quantitation for minimal residual disease monitoring in childhood AML and implications of WT1 gene mutations: a European multicenter study. Leukemia. 2009; 23:1472-1479.

13. Coustan-Smith E, Sancho J, Behm FG, Hancock ML, Razzouk BI, Ribeiro RC, Rivera GK, Rubnitz JE, Sandlund JT, Pui CH, Campana D. Prognostic importance of measuring early clearance of leukemic cells by flow cytometry in childhood acute lymphoblastic leukemia. Blood. 2002;100:52-58.
14. Venditti A, Buccisano F, Del Poeta G, Maurillo L, Tamburini A, Cox C, Battaglia A, Catalano G, Del Moro B, Cudillo L, Postorino M, Masi M, Amadori S. Level of minimal residual disease after consolidation therapy predicts outcome in acute myeloid leukemia. Blood. 2000; 96:3948-3952.

15. Stern M, Ruggeri L, Mancusi A, Bernardo ME, de Angelis C, Bucher C, Locatelli F, Aversa F, Velardi A. Survival after $\mathrm{T}$ cell-depleted haploidentical stem cell transplantation is improved using the mother as donor. Blood. 2008;112:2990-2995.

16. Horowitz MM, Rowlings PA. An update from the International Bone Marrow Transplant Registry and the Autologous Blood and Marrow Transplant Registry on current activity in hematopoietic stem cell transplantation. Current opinion in hematology. 1997;4:395-400.

17. Sierra J, Storer B, Hansen JA, Martin PJ, Petersdorf EW, Woolfrey A, Matthews D, Sanders JE, Storb R, Appelbaum FR, Anasetti C. Unrelated donor marrow transplantation for acute myeloid leukemia: an update of the Seattle experience. Bone marrow transplantation. 2000;26:397-404.

18. Tauro S, Craddock C, Peggs K, Begum G, Mahendra P, Cook G, Marsh J, Milligan D, Goldstone A, Hunter A, Khwaja A, Chopra R, Littlewood T, et al. Allogeneic stemcell transplantation using a reduced-intensity conditioning regimen has the capacity to produce durable remissions and long-term disease-free survival in patients with high-risk acute myeloid leukemia and myelodysplasia. Journal of Clinical Oncology. 2005;23:9387-9393.

19. Locke F, Artz A, Rich E, Zhang Y, van Besien K, Stock W. Feasibility of clofarabine cytoreduction before allogeneic transplant conditioning for refractory AML. Bone marrow transplantation. 2010;45:1692-1698.

20. Mehta DR, Foon KA, Redner RL, Raptis A, Agha M, Hou JZ, Duggal S, Luong TM, Schlesselman JJ, Boyiadzis M. Fludarabine and cytarabine in patients with acute myeloid leukemia refractory to two different courses of front-line chemotherapy. Leukemia research. 2011;35:885-888.

21. McLaughlin B, Im A, Raptis A, Agha M, Hou JZ, Redner R, Duggal S, Lin Y, Smith C, Boyiadzis M. Fludarabine and cytarabine in patients with relapsed acute myeloid leukemia refractory to initial salvage therapy. International journal of hematology. 2012;96:743-747.

22. Kebriaei P, Kline J, Stock W, Kasza K, Le Beau MM, Larson RA, van Besien K. Impact of disease burden at time of allogeneic stem cell transplantation in adults with acute myeloid leukemia and myelodysplastic syndromes. Bone marrow transplantation. 2005;35:965-970.

23. van Besien K, Artz A, Smith S, Cao D, Rich S, Godley L, Jones D, Del Cerro P, Bennett D, Casey B, Odenike $\mathrm{O}$, Thirman $\mathrm{M}$, Daugherty $\mathrm{C}$, et al. Fludarabine, melphalan, and alemtuzumab conditioning in adults with standard-risk advanced acute myeloid leukemia and 
myelodysplastic syndrome. Journal of clinical oncology. 2005;23:5728-5738.

24. Oran B, Giralt S, Saliba R, Hosing C, Popat U, Khouri I, Couriel D, Qazilbash M, Anderlini P, Kebriaei P, Ghosh S, Carrasco-Yalan A, de Meis E, et al. Allogeneic hematopoietic stem cell transplantation for the treatment of high-risk acute myelogenous leukemia and myelodysplastic syndrome using reduced-intensity conditioning with fludarabine and melphalan. Biology of Blood and Marrow Transplantation. 2007;13:454-462.

25. Kasamon YL, Luznik L, Leffell MS, Kowalski J, Tsai HL, Bolaños-Meade J, Morris LE, Crilley PA, O’Donnell PV, Rossiter N, Huff CA, Brodsky RA, Matsui WH, et al. Nonmyeloablative HLA-haploidentical bone marrow transplantation with high-dose posttransplantation cyclophosphamide: effect of HLA disparity on outcome. Biology of Blood and Marrow Transplantation. 2010;16:482-489.

26. Wang Y, Chang YJ, Xu LP, Liu KY, Liu DH, Zhang $\mathrm{XH}$, Chen H, Han W, Chen YH, Wang FR, Wang JZ, Chen $\mathrm{Y}$, Yan $\mathrm{CH}$, et al. Who is the best donor for a related HLA-haplotype-mismatched transplant? Blood. 2014;124:843-850.

27. Johansson J, Brune M, Ekman T. The gut mucosa barrier is preserved during allogeneic, haemopoietic stem cell transplantation with reduced intensity conditioning. Bone marrow transplantation. 2001;28:737-742.

28. Niscola P, Romani C, Cupelli L, Scaramucci L, Tendas A, Dentamaro T, Amadori S, de Fabritiis P. Mucositis in patients with hematologic malignancies: an overview. Haematologica. 2007;92:222-231.
29. Network NCCN. NCCN Practice Guidelines in Oncology. Version 1.2016; 2016.

30. Gratwohl A. The EBMT risk score. Bone marrow transplantation. 2012;47:749-756.

31. Schmid C, Schleuning M, Ledderose G, Tischer J, Kolb H-J. Sequential regimen of chemotherapy, reduced-intensity conditioning for allogeneic stem-cell transplantation, and prophylactic donor lymphocyte transfusion in high-risk acute myeloid leukemia and myelodysplastic syndrome. Journal of Clinical Oncology. 2005;23:5675-5687.

32. Glucksberg H, Storb R, Fefer A, Buckner CD, Neiman PE, Clift RA, Lerner KG, Thomas ED. Clinical manifestations of graft-versus-host disease in human recipients of marrow from HLA-matched sibling donor. Transplantation. 1974;18:295-304.

33. Lee SJ, Vogelsang G, Flowers ME. Chronic graft-versushost disease. Biology of Blood and Marrow Transplantation. 2003;9:215-233.

34. Health UDo, Services H. Common terminology criteria for adverse events (CTCAE) version 4.0. National Institutes of Health, National Cancer Institute. 2009;4.

35. Health UDo, Services H. Common terminology criteria for adverse events: Version; 2014.

36. Bader P, Beck J, Frey A, Schlegel PG, Hebarth H, Handgretinger R, Einsele H, Niemeyer C, Benda N, Faul C, Kanz L, Niethammer D, Klingebiel T. Serial and quantitative analysis of mixed hematopoietic chimerism by PCR in patients with acute leukemias allows the prediction of relapse after allogeneic BMT. Bone marrow transplantation. 1998;21:487-495. 